\title{
Sensores de reflectância e fluorescência na avaliação de teores de nitrogênio, produção de biomassa e produtividade do algodoeiro
}

\author{
Otavio Bagiotto Rossato(1), Pedro Andrade-Sanchez ${ }^{(2)}$, Saulo Philipe Sebastião Guerra(1) \\ e Carlos Alexandre Costa Crusciol(1)
}

\begin{abstract}
(1)Universidade Estadual Paulista, Faculdade de Ciências Agronômicas, Rua José Barbosa de Barros, no 1.780, Caixa Postal 237,

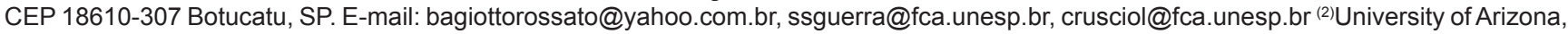
Maricopa Agricultural Center, № 37.860 W., Smith-Enke Road, AZ 85239-3010, Maricopa, AZ, EUA. E-mail: pandrade@ag.arizona.edu
\end{abstract}

Resumo - O objetivo deste trabalho foi avaliar o potencial de utilização dos sensores de reflectância e fluorescência na avaliação de teores de $\mathrm{N}_{-} \mathrm{NO}_{3}{ }^{-}$no pecíolo, na produção de biomassa e na produtividade do algodoeiro. Utilizou-se o delineamento experimental de blocos ao acaso em arranjo fatorial $3 \times 4$, com quatro repetições. Os tratamentos consistiram de três variedades de algodão (ST-4288-B2RF, ST-4498-B2RF e DP164-B2RF) e quatro doses de $\mathrm{N}\left(0,45,90\right.$ e $\left.135 \mathrm{~kg} \mathrm{ha}^{-1}\right)$. Aos 120 dias após a semeadura, foram realizadas leituras com sensores ópticos de reflectância e fluorescência. Não houve correlação significativa dos teores de $\mathrm{N}_{-} \mathrm{NO}_{3}{ }^{-}$no pecíolo com os índices de reflectância; porém, houve correlação destes com a produção de biomassa $(0,39)$ e a produtividade $(0,32$ a 0,41$)$. Os índices do sensor de fluorescência correlacionaram-se significativamente com teores de $\mathrm{N}_{-} \mathrm{NO}_{3}{ }^{-}$no pecíolo $(0,34$ a 0,61$)$, produção de biomassa $(0,30$ a 0,53$)$ e produtividade $(0,34)$. Em comparação com os índices de reflectância, os de fluorescência apresentam maior capacidade de avaliar os teores de $\mathrm{N}_{-} \mathrm{NO}_{3}{ }^{-}$no pecíolo, capacidade semelhante em detectar variação na biomassa e menor capacidade de detectar variação da produtividade do algodoeiro, quando se aplicam doses crescentes de N.

Termos para indexação: Gossypium, agricultura de precisão, estado nutricional, índices de vegetação, NDVI, sensoriamento remoto.

\section{Reflectance and fluorescence sensors to assess nitrogen levels, biomass production and yield of cotton}

\begin{abstract}
The objective of this work was to evaluate the potential of using reflectance and fluorescence sensors

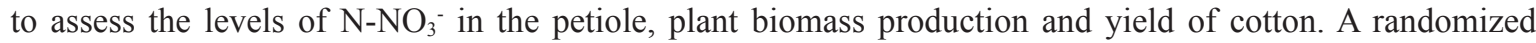
complete block design was used in a $3 \times 4$ factorial arrangement, with four replicates. Treatments consisted of three cotton varieties (ST-4288-B2RF, ST-4498-B2RF and DP-164-B2RF) and of four N rates $(0,45,90$ and $\left.135 \mathrm{~kg} \mathrm{ha}^{-1}\right)$. At 120 days after sowing, readings were done with optical sensors for canopy fluorescence and reflectance. There were no significant correlations for $\mathrm{N}_{-} \mathrm{NO}_{3}$ in the petiole with the reflectance sensor indices; however, there was correlation to biomass production ( 0.39$)$ and yield $(0.32$ to 0.41$)$. The fluorescence sensor indices were significantly correlated to $\mathrm{N}^{-\mathrm{NO}_{3}}$ in the petiole $(0.34$ to 0.61$)$, biomass production $(0.30$ to 0.53$)$ and yield (0.34). Compared to the reflectance indices, the fluorescence ones have a greater ability to assess the levels of $\mathrm{N}^{-\mathrm{NO}_{3}}{ }^{-}$in the petiole, a similar ability to detect variation of plant biomass, and a lower ability to detect the variation in cotton yield when increasing rates of nitrogen are applied.
\end{abstract}

Index terms: Gossypium, precision agriculture, nutritional status, vegetation indices, NDVI, remote sensing.

\section{Introdução}

A deficiência e o excesso de $\mathrm{N}$ afetam negativamente o crescimento das plantas de algodão, a retenção de capulhos, a produtividade e a qualidade das fibras (Reddy et al., 2004). A variabilidade espacial da fertilidade do solo e a variabilidade na exigência de $\mathrm{N}$ durante diferentes fases de crescimento de algodão são razões adicionais para avaliar o estado nutricional do algodão quanto a esse nutriente.
A aplicação de fertilizantes a taxas variáveis pode aumentar sua eficiência, em comparação à aplicação de uma dose fixa. Para implementar a aplicação de acordo com a necessidade das plantas, é fundamental que se monitore os níveis de suficiência de $\mathrm{N}$. O método convencional de monitoramento de $\mathrm{N}$ no algodão atualmente inclui amostragem e análise de tecidos do pecíolo (Henslee et al., 2002) e das folhas (Malavolta et al., 1997). No entanto, alternativas para a detecção 
rápida e não destrutiva do estado nutricional de $\mathrm{N}$ nas plantas têm sido objetivo de muitas pesquisas.

Dados de sensoriamento remoto são amplamente utilizados para desenvolver índices de vegetação como indicadores de crescimento da cultura, do estado nutricional e da produtividade. Vários autores têm sugerido que a reflectância espectral de culturas pode ser usada para detectar estresses bióticos e abióticos do ambiente (Osborne et al., 2002), avaliar o estado nutricional (Zhao et al., 2005), estimar o crescimento (Zhao et al., 2003) e monitorar as condições da planta, bem como para prever a produtividade das culturas (Ma et al., 2001). A espectrometria óptica é baseada na premissa de que pigmentos como a clorofila absorvem a radiação nos comprimentos de onda dentro do espectro do visível e refletem a radiação no infravermelho próximo (Minolta, 1989).

Por meio da determinação dos teores de clorofila, pode-se obter uma estimativa indireta do estado nutricional da planta quanto ao $\mathrm{N}$, pois grande parte do que as plantas absorvem desse nutriente é utilizado para a síntese dessa molécula (Rambo et al., 2004). Dessa forma, muitas pesquisas têm sido feitas para desenvolver algoritmos que traduzam o valor fornecido pelo sensor em decisões sobre o quanto aplicar de $\mathrm{N}$ nas culturas (Raun et al., 2005; Ramirez, 2010; Solari et al., 2010).

O uso de medidas de fluorescência como indicador do estado nutricional das culturas, quanto ao N, ainda está em fase inicial em relação a outros métodos de diagnose nutricional. Goulas et al. (2004) desenvolveram um dispositivo portátil, o Dualex (Force-A, Orsay, França), que pode avaliar o estado nutricional em $\mathrm{N}$ por meio da detecção do conteúdo de polifenóis da folha. Já o Multiplex (Force-A, Orsay, França) é um sensor óptico que gera fluorescência nos tecidos da planta com uso de fontes múltiplas de excitação de luz (ultravioleta, azul, verde e vermelho), para estimar simultaneamente os conteúdos de vários compostos, tais como os de antocianina, flavonoides e clorofila.

$\mathrm{O}$ uso de medidas de reflectância e fluorescência na estimativa da biomassa de plantas de algodão pode ajudar os produtores quanto a decisões de manejo, como irrigação, adubação foliar e aplicação de reguladores de crescimento para obtenção de produtividade máxima (Zhao et al., 2007).

Apesar da abundância de pesquisas com sensores de reflectância, principalmente em culturas como o milho e o trigo, são escassos os trabalhos com sensores de fluorescência, e há carência de informação sobre como este sensor deve ser usado no campo ou qual sua sensibilidade em estimar medidas agronômicas.

O objetivo do presente trabalho foi avaliar o potencial de utilização dos índices fornecidos por sensores de reflectância e fluorescência na avaliação dos teores de $\mathrm{N}^{-\mathrm{NO}_{3}}$ no pecíolo, produção de biomassa e produtividade do algodoeiro.

\section{Material e Métodos}

Os sensores ópticos de reflectância e fluorescência foram testados em algodoeiro, em área pertencente à Universidade do Arizona (a 330" $\mathrm{N}, 111^{\circ} 58^{\circ} \mathrm{W}, 361 \mathrm{~m}$ de altitude), junto ao Maricopa Center Agriculture, Maricopa, AZ, EUA. A área experimental, com solo de textura franco-arenosa (71\% areia, $12 \%$ argila e $17 \%$ silte), foi irrigada por sulcos.

Antes da semeadura, determinou-se o teor médio de nitrato da água de irrigação, que foi de 1,25 ppm. Além disso, a quantidade de $\mathrm{N}^{-\mathrm{NO}_{3}}$ contida no solo, antes da implantação do experimento, era de aproximadamente $45 \mathrm{~kg} \mathrm{ha}^{-1}$ de N.

Empregou-se o delineamento experimental de blocos ao acaso, em arranjo fatorial $3 \times 4$, com quatro repetições. Os tratamentos consistiram das variedades de algodão (Gossypium hirsutum L.) ST-4288-B2RF, ST-4498-B2RF e DP-164-B2RF e das doses de 0, 45, 90 e $135 \mathrm{~kg} \mathrm{ha}^{-1}$ de $\mathrm{N}$.

O algodão foi semeado no dia 23 de abril de 2010, em espaçamento de $1,0 \mathrm{~m}$ e à densidade de 122 mil plantas por hectare. No dia 14 de julho de 2010, realizou-se aplicação de $\mathrm{N}$ na forma de nitrato de amônio. Os demais tratos culturais foram realizados de acordo com as necessidades $\mathrm{e}$ as recomendações técnicas para a cultura.

O conteúdo de nitrato no pecíolo do algodoeiro foi determinado aos 120 dias após a semeadura. Os pecíolos foram coletados em 25 folhas por parcela, na primeira folha totalmente expandida $(+1)$. Após a coleta, o material foi levado para a estufa, seco a $70^{\circ} \mathrm{C}$ até massa constante, e o conteúdo de nitrato foi determinado.

As análises de solo e planta foram realizadas pelo método do $\mathrm{N}_{-} \mathrm{NO}_{3}^{-}$via redução de $\mathrm{Cd}$, método modificado de Griess-Ilsovay, por meio do Alpkem modelo 320 RFA II (Alpkem Corp., College Station, TX, EUA) tendo-se seguido metodologia proposta por Miller \& Kotuby-Amacher (1994). 
A determinação da biomassa foi realizada com a coleta das plantas em $1 \mathrm{~m}^{2}$, aos 120 dias após a semeadura. Após a coleta, o material foi levado para a estufa e seco a $70^{\circ} \mathrm{C}$ até atingir massa constante. Aos 180 dias após a semeadura, foi realizada a colheita mecânica de $20 \mathrm{~m}^{2}$, com posterior pesagem, para determinação da produtividade final de algodão.

A coleta de dados com os sensores de reflectância e fluorescênciafoirealizadaaos 120 dias após a semeadura, no estádio de emissão do primeiro capulho. Os dados de reflectância no algodoeiro foram coletados por quatro sensores de reflectância Crop Circle modelo ACS-470, instalados na frente de um trator, e o datalloger, dentro da cabine do trator, registrou esses dados de reflectância das plantas de algodão. Foram feitas 36 leituras por parcela. O sensor de reflectância Crop Circle fornece dados em três bandas: 670, 720 e $820 \mathrm{~nm}$. A partir desses dados, foram calculados os índices: NDVI $=(\rho 820 \mathrm{~nm}-\rho 670 \mathrm{~nm}) /(\rho 820 \mathrm{~nm}+\rho 670 \mathrm{~nm})$, e de clorofila $=(\rho 820 \mathrm{~nm} / \rho 720 \mathrm{~nm})-1$, em que $\rho$ é a reflectância.

O Multiplex 3.0 (FORCE-A, Orsay, França) possui diodos emissores de luz (LED), dos quais seis são de ultravioleta (UV) e três de vermelho, verde, azul (RGB), e três detectores de fluorescência: amarelo (YF), de $590 \mathrm{~nm}$; vermelho (RF), de $685 \mathrm{~nm}$; e vermelho extremo (FRF), de $735 \mathrm{~nm}$. Com esse sensor de fluorescência, foram coletados manualmente 12 pontos por parcela, sobre as plantas na linha de cultivo do algodoeiro. Os índices fornecidos pelo sensor de fluorescência estão descritos na Tabela 1 .

Os dados foram submetidos à análise de variância e, quando os efeitos foram significativos, utilizou-se o teste de Tukey para comparação de médias entre variedades ou ajustaram-se equações de regressão para avaliação do fator doses de $\mathrm{N}$, tendo-se por base os níveis de significância de $5 \%$ de probabilidade. A correlação de Pearson (r) entre os índices e as variáveis também foi avaliada.

Para determinar a dose de máxima produtividade, utilizou-se a equação ajustada entre produtividade e doses de N. Para determinar a máxima eficiência econômica (MEE), igualou-se a derivada primeira da equação de regressão, correspondente à produtividade no valor zero, e a relação entre preços do insumo (US\$ por $\mathrm{kg}$ de $\mathrm{N}$, na forma de nitrato de amônio) e do produto (US\$ por $\mathrm{kg}$ de algodão em caroço) (Raij, 1991), tendo-se considerado os preços vigentes no Estado do Arizona, EUA, em março de 2010 (United States Department of Agriculture, 2010). A fórmula obtida para determinação da dose de máxima eficiência econômica foi: dose de $\mathrm{N}=(4,7985$ - Y $) /(2 \times 0,0284)$, em que Y é a relação entre os preços do insumo e do produto.

O valor ideal de cada índice, no estádio de emissão do primeiro capulho, foi determinado por meio das equações de regressão ajustadas para a dose de MEE.

\section{Resultados e Discussão}

Em todos os índices e parâmetros avaliados, não houve interação entre as variedades de algodão e doses de $\mathrm{N}$ (Tabela 2). Em relação às doses de $\mathrm{N}$, observouse que elas afetaram significativamente: as leituras dos índices NDVI, CLR, YF_UV, RF_UV, FRF_UV, BRR_FRF,FER_RUV,FLAV,ANTH, NBI_GeNBI_R; os teores de N no pecíolo; a produção de biomassa; e a produtividade do algodoeiro. Observou-se também que as variedades de algodão afetaram significativamente a produtividade e as leituras dos índices NDVI, CLR, YF_B, RF_B, YF_G, RF_G, FRF_G e ANTH. Porém, as variedades não influenciaram as leituras dos demais índices do sensor de fluorescência, o que indica que

Tabela 1. Descrição dos índices fornecidos pelo sensor de fluorescência Multiplex 3 (Force A, 2010).

\begin{tabular}{llcc}
\hline Indices & \multicolumn{1}{c}{ Descrição } & Excitação & Fórmula \\
\hline YF_UV & Fluorescência amarela & UV & - \\
RF_UV & Fluorescência vermelha & UV & - \\
FRF_UV & Fluorescência vermelho distante & UV & - \\
YF_B & Fluorescência amarela & B & - \\
RF_B & Fluorescência vermelha & B & - \\
FRF_B & Fluorescência vermelho distante & B & - \\
YF_G & Fluorescência amarela & G & - \\
RF_G & Fluorescência vermelha & G & - \\
FRF_G & Fluorescência vermelho distante & G & - \\
YF_R & Fluorescência amarela & R & - \\
RF_R & Fluorescência vermelha & R & - \\
FRF_R & Fluorescência vermelho distante & R & - \\
SFR_G & Simples relação de fluorescência & G & FRF_G/RF_G \\
SFR_R & Simples relação de fluorescência & R & FRF_R/RF_R \\
BRR_FRF & Fluorescência azul/vermelho & UV & YF_UV/FRF_UV \\
FER_RUV Fluorescência do vermelho/UV & R \& UV & FRF_R/FRF_UV \\
FLAV & Flavonoides & R \& UV & Log(FRF_R/FRF_UV) \\
FER_RG & Fluorescência do vermelho/verde & R \& G & FRF_R/FRF_G \\
ANTH & Antocianinas & R \& G & Log(FRF_R/FRF_G) \\
NBI_G & Índice - balanço de N & UV \& G & FRF_UV/RF_G \\
NBI_R & Índice - balanço de N $\&$ R & FRF_UV/RF_R \\
FERARI & Taxa de excitação da fluorescência & R & Log(5000/FRF_R) \\
\hline
\end{tabular}

UV, ultravioleta; B, azul; G, verde; R, vermelho. 
estes são menos afetados no estádio de emissão do primeiro capulho.

Em relação ao índice NDVI, verificou-se que a variedade ST-4498-B2RF apresentou valores superiores em comparação às demais variedades (Tabela 3). Quanto aos índices fornecidos pelo sensor de fluorescência (RF_B, FRF_G e ANTH) a variedade ST-4288-B2RF diferiu das variedades ST-4498-B2RF e DP-164-B2RF. A clorofila mensurada pelo sensor de reflectância foi a única variável capaz de diferenciar as três variedades de algodão. No entanto, se este fato mostra a capacidade dos sensores de detectar diferentes variedades, mostra também a necessidade da calibração do sensor para o uso em diferentes variedades. Fontana et al. (1998) relatam que as mudanças estruturais na vegetação, no decorrer da estação de crescimento, resultam em uma diferenciação da sua reflectância, o que permite empregar o NDVI para o monitoramento da vegetação, bem como para distinguir diferentes

Tabela 2. Análise da variância dos índices dos sensores de reflectância e fluorescência em três variedades de algodão.

\begin{tabular}{|c|c|c|c|c|c|}
\hline \multirow{2}{*}{$\begin{array}{l}\text { Causas de } \\
\text { variação }^{(1)}\end{array}$} & \multicolumn{3}{|c|}{ Quadrados Médios } & \multirow[t]{2}{*}{ Média } & \multirow{2}{*}{$\begin{array}{l}\mathrm{CV} \\
(\%)\end{array}$} \\
\hline & Variedades & Doses de N & Var $\times \mathrm{N}$ & & \\
\hline NDVI & $0,00158 * *$ & $0,00018^{*}$ & $0,00003^{\mathrm{ns}}$ & 0,819 & 1,05 \\
\hline CLR & $0,2111^{* *}$ & $0,0549 * *$ & $0,0025^{\text {ns }}$ & 2,69 & 2,42 \\
\hline YF_UV & $0,1416^{\mathrm{ns}}$ & $0,0029^{\text {ns }}$ & $0,0257^{\mathrm{ns}}$ & 5,93 & 5,08 \\
\hline RF_UV & $4,61^{\mathrm{ns}}$ & $119,08^{* *}$ & $6,44^{\mathrm{ns}}$ & 30,55 & 8,91 \\
\hline FRF_UV & $39,53^{\text {ns }}$ & $3549,21 * *$ & $131,39^{\text {ns }}$ & 147,7 & 11,44 \\
\hline YF_B & $18,7^{*}$ & $5,12^{\mathrm{ns}}$ & $1,72^{\mathrm{ns}}$ & 17,2 & 13,59 \\
\hline RF_B & $18937^{*}$ & $5660^{\mathrm{ns}}$ & $2257^{\mathrm{ns}}$ & 594 & 11,1 \\
\hline FRF_B & $258365^{\mathrm{ns}}$ & $170058^{\mathrm{ns}}$ & $121862^{\mathrm{ns}}$ & 2934 & 10,91 \\
\hline YF_G & $50451^{*}$ & $12709^{\mathrm{ns}}$ & $6103^{\text {ns }}$ & 704 & 16,35 \\
\hline RF_G & $2097 * *$ & $171^{\mathrm{ns}}$ & $223^{\mathrm{ns}}$ & 201 & 11,98 \\
\hline FRF_G & $261602 * *$ & $15232^{\text {ns }}$ & $20373^{\mathrm{ns}}$ & 1913 & 9,91 \\
\hline YF_R & $2545^{\text {ns }}$ & $2725^{\text {ns }}$ & $699^{\mathrm{ns}}$ & 207 & 16,29 \\
\hline RF_R & $1342^{\text {ns }}$ & $755^{\mathrm{ns}}$ & $718^{\mathrm{ns}}$ & 300 & 13,21 \\
\hline FRF_R & $193879^{\mathrm{ns}}$ & $98087^{\mathrm{ns}}$ & $78176^{\mathrm{ns}}$ & 2612 & 12,16 \\
\hline SFR_G & $0,4738^{\text {ns }}$ & $0,2549^{\text {ns }}$ & $0,2183^{\mathrm{ns}}$ & 9,56 & 8,75 \\
\hline SFR_R & $0,5704^{\mathrm{ns}}$ & $0,4172^{\text {ns }}$ & $0,4074^{\mathrm{ns}}$ & 8,74 & 10,6 \\
\hline BRR_FRF & $0,000014^{\mathrm{ns}}$ & $0,00031 * *$ & $0,000023^{\mathrm{ns}}$ & 0,0410 & 11,65 \\
\hline FER_RUV & $15,54^{\mathrm{ns}}$ & $85,22 * *$ & $10,38^{\mathrm{ns}}$ & 18,10 & 15,72 \\
\hline FLAV & $0,0087^{\mathrm{ns}}$ & $0,0485^{* *}$ & $0,0042^{\mathrm{ns}}$ & 1,249 & 5,62 \\
\hline FER_RG & $0,0238^{\mathrm{ns}}$ & $0,0271^{\mathrm{ns}}$ & $0,0021^{\mathrm{ns}}$ & 1,37 & 6,37 \\
\hline ANTH & $0,0025^{\mathrm{ns}}$ & $0,0028^{*}$ & $0,0002^{\mathrm{ns}}$ & 0,13 & 20,63 \\
\hline NBI_G & $0,0329^{\text {ns }}$ & $0,1039 * *$ & $0,0058^{\mathrm{ns}}$ & 0,75 & 16,01 \\
\hline NBI_R & $0,0039^{\mathrm{ns}}$ & $0,0594 * *$ & $0,0029^{\text {ns }}$ & 0,503 & 19,9 \\
\hline FERARI & $0,0061^{\mathrm{ns}}$ & $0,0025^{\text {ns }}$ & $0,0023^{\mathrm{ns}}$ & 0,285 & 18,85 \\
\hline $\mathrm{N}-\mathrm{NO}_{3}^{-}$pecíolo & $3467^{\mathrm{ns}}$ & $97379 * *$ & $3046^{\mathrm{ns}}$ & 9,22 & 20,07 \\
\hline Biomassa & $23240^{\mathrm{ns}}$ & $26731^{*}$ & $1262^{\mathrm{ns}}$ & 565,4 & 16,34 \\
\hline Produtividade & $846085^{* *}$ & $104152 *$ & $78174^{\text {ns }}$ & 4943 & 3,62 \\
\hline
\end{tabular}

${ }^{(1)}$ Tabela $1 .{ }^{\text {ns }}$ Não significativo. * e**Significativo a 5 e a $1 \%$ de probabilidade, respectivamente, pelo teste $\mathrm{F}$. tipos de vegetação e detectar possíveis problemas de crescimento.

De acordo com Ramirez (2010), é comum que as variedades de algodoeiro influenciem as leituras de sensores de reflectância. Schächtl et al. (2005), por outro lado, relatam que as leituras de fluorescência também são influenciadas pelas variedades de trigo.

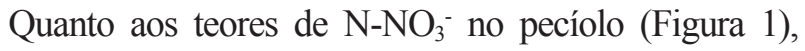
observou-se resposta quadrática à aplicação de $\mathrm{N}$, em que o incremento nos teores foi praticamente nulo, a partir de $90 \mathrm{~kg} \mathrm{ha}^{-1}$ de $\mathrm{N}$, e atingiu o máximo quando foram aplicados $110 \mathrm{~kg} \mathrm{ha}^{-1}$ de N. Segundo Pennington \& Tucker (1984), os teores de $\mathrm{N}^{-\mathrm{NO}_{3}}{ }_{3}^{-}$no pecíolo durante a emissão dos primeiros capulhos devem estar acima de $8 \mathrm{~g} \mathrm{~kg}^{-1}$ para serem considerados adequados. No presente estudo, este valor foi superior ao recomendado já na menor dose, de $45 \mathrm{~kg} \mathrm{ha}^{-1}$ de N. Isto, provavelmente, ocorreu em razão de que, além de haver $\mathrm{N}$ na água de irrigação, o solo continha, antes da semeadura, $45 \mathrm{~kg} \mathrm{ha}^{-1} \mathrm{de} \mathrm{N}$, o que pode ter suprido grande parte das necessidades de $\mathrm{N}$ requeridas pelo algodão.

A máxima produção de biomassa (Figura 1) ocorreu com a aplicação da maior dose de $\mathrm{N}\left(135 \mathrm{~kg} \mathrm{ha}^{-1}\right)$; porém, isso não se refletiu em máxima produtividade, que foi atingida com a dose de $84 \mathrm{~kg} \mathrm{ha}^{-1}$. Segundo Staut \& Kurihara (2001), quando Né aplicado em doses elevadas, o desenvolvimento vegetativo da planta aumenta em detrimento da produção e formação tardia dos frutos do algodoeiro. Além disso, Cisneros \& Godfrey (2001) salientam que o excesso de $\mathrm{N}$ pode afetar indiretamente a qualidade das fibras, pelo aumento da infestação de pulgões.

Nas correlações entre os índices do sensor de fluorescência e os índices do sensor de reflectância,

Tabela 3. Desdobramento do fator variedade para os índices dos sensores de reflectância e fluorescência, em três variedades de algodão(1).

\begin{tabular}{lcccc}
\hline Causa de variação & & \\
\hline NDVI & ST 4288-B2RF & ST 4288-B2RF & DP 164-B2RF & CV (\%) \\
CLR & $0,811 \mathrm{~b}$ & $0,830 \mathrm{a}$ & $0,816 \mathrm{~b}$ & 0,88 \\
YF_B & $2,80 \mathrm{a}$ & $2,69 \mathrm{~b}$ & $2,57 \mathrm{c}$ & 2,44 \\
RF_B & $16,2 \mathrm{~b}$ & $18,3 \mathrm{a}$ & $17,1 \mathrm{ab}$ & 13,26 \\
YF_G & $554 \mathrm{~b}$ & $612 \mathrm{a}$ & $615 \mathrm{a}$ & 11,54 \\
RF_G & $642 \mathrm{~b}$ & $752 \mathrm{a}$ & $717 \mathrm{ab}$ & 16,23 \\
FRF_G & $188 \mathrm{~b}$ & $210 \mathrm{a}$ & $205 \mathrm{ab}$ & 11,81 \\
FER_RG & $1766 \mathrm{~b}$ & $1990 \mathrm{a}$ & $1984 \mathrm{a}$ & 10,25 \\
ANTH & $1,41 \mathrm{a}$ & $1,36 \mathrm{~b}$ & $1,33 \mathrm{~b}$ & 4,65 \\
Produtividade $\left(\mathrm{kg} \mathrm{ha}^{-1}\right)$ & $0,15 \mathrm{a}$ & $0,13 \mathrm{~b}$ & $0,12 \mathrm{~b}$ & 15,34 \\
\hline
\end{tabular}

${ }^{(1)}$ Médias seguidas de letras iguais, nas linhas, não diferem entre si pelo teste de Tukey, a 5\% de probabilidade. ${ }^{(2)}$ Tabela 1. 
observou-se que o NDVI apresentou correlações significativas com os índices FER_RG e ANTH (Tabela 4), e que a clorofila correlacionou-se significativamente com RF_UV, FRF_UV, FRF_G,
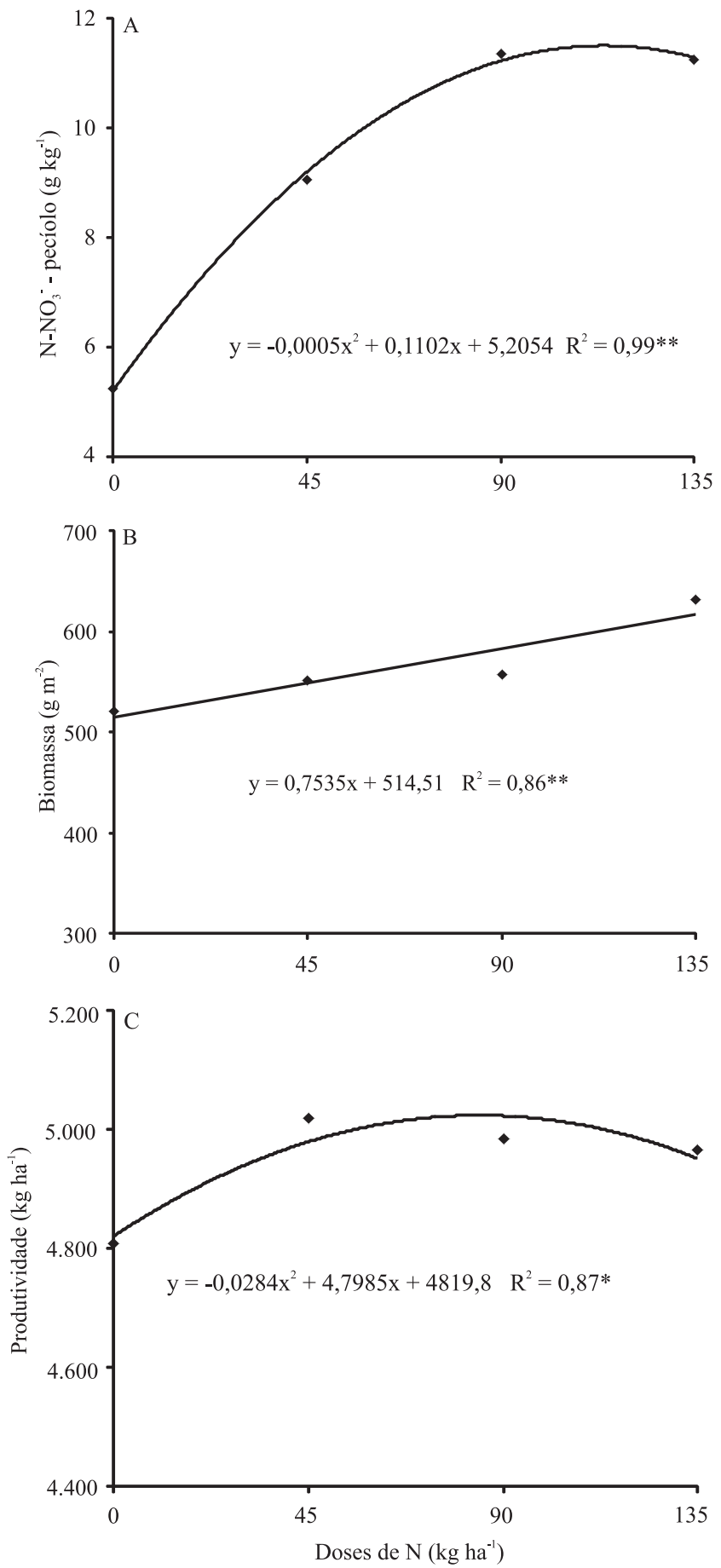

Figura 1. Efeito de doses de $\mathrm{N}$ sobre: $\mathrm{A}$, teor de $\mathrm{N}^{-\mathrm{NO}_{3}}{ }^{-}$ no pecíolo; $\mathrm{B}$, produção de biomassa; e $\mathrm{C}$, produtividade do algodoeiro.
BRR FRF, FER RUV, FLAV e NBI G. A partir dessas correlações, pode-se inferir que estes índices apresentam comportamento similar e que, na ausência de um desses sensores, é possível estimar os índices do outro sem que ocorra perda significativa de informação.

Os índices fornecidos pelo sensor de reflectância NDVI e clorofila (Tabela 4) não se correlacionaram com os teores de $\mathrm{N}_{-} \mathrm{NO}_{3}^{-}$no pecíolo, a partir de leituras realizadas no estádio de emissão do primeiro capulho. Esses dados concordam com os de Bronson et al. (2005), que relataram que o NDVI medido com o sensor de reflectância, aos 75 dias após a semeadura, esteve pouco correlacionado com $\mathrm{N}$ foliar do algodoeiro. No entanto, Motomiya et al. (2009) observaram relação linear simples entre o $\mathrm{N}$ nas folhas do algodoeiro e o índice NDVI $(0,61)$, aos 92 dias após a emergência de plantas de algodão. As baixas correlações entre os índices fornecidos pelos sensores de reflectância e os teores de $\mathrm{N}$ provavelmente estão associadas à saturação ou "consumo de luxo" de $\mathrm{N}$ pelas plantas nas doses mais elevadas, uma vez que o $\mathrm{N}$ não incorporado às

Tabela 4. Correlação entre os índices dos sensores de reflectância e fluorescência e os teores de $\mathrm{N}-\mathrm{NO}_{\overline{3}}$ no pecíolo, biomassa e produtividade de plantas de algodão.

\begin{tabular}{|c|c|c|c|c|c|}
\hline Îndice $^{(1)}$ & NDVI & Clorofila & Biomassa & $\mathrm{N}-\mathrm{NO}_{3}^{-}$pecíolo & Produtividade \\
\hline$\overline{\text { NDVI }}$ & 1,00 & - & $0,39 *$ & $0,21^{\mathrm{ns}}$ & $0,32 *$ \\
\hline Clorofila & $0,07^{\mathrm{ns}}$ & 1,00 & $0,21^{\mathrm{ns}}$ & $0,27^{\mathrm{ns}}$ & $0,41^{*}$ \\
\hline YF_UV & $0,19^{\mathrm{ns}}$ & $0,07 \mathrm{~ns}$ & $-0,09^{\text {ns }}$ & $-0,14^{\mathrm{ns}}$ & $0,34 *$ \\
\hline RF_UV & $0,22^{\text {ns }}$ & $0,38^{*}$ & $0,26^{\mathrm{ns}}$ & $0,57^{*}$ & $0,18^{\mathrm{ns}}$ \\
\hline FRF_UV & $0,28^{\mathrm{ns}}$ & $0,31 *$ & $0,39^{*}$ & $0,54 *$ & $0,25^{\mathrm{ns}}$ \\
\hline YF_B & $0,18^{\mathrm{ns}}$ & $-0,18^{\text {ns }}$ & $-0,15^{\text {ns }}$ & $-0,06^{\text {ns }}$ & $-0,13^{\text {ns }}$ \\
\hline RF_B & $0,14^{\mathrm{ns}}$ & $-0,18^{\text {ns }}$ & $-0,16^{\mathrm{ns}}$ & $0,34 *$ & $-0,04^{\text {ns }}$ \\
\hline FRF_B & $0,10^{\mathrm{ns}}$ & $-0,07^{\text {ns }}$ & $-0,18^{\mathrm{ns}}$ & $0,21^{\mathrm{ns}}$ & $0,02^{\mathrm{ns}}$ \\
\hline YF_G & $0,21^{\mathrm{ns}}$ & $-0,26^{\text {ns }}$ & $-0,22^{\mathrm{ns}}$ & $-0,11^{\text {ns }}$ & $-0,17^{\mathrm{ns}}$ \\
\hline RF_G & $0,15^{\text {ns }}$ & $-0,19^{\text {ns }}$ & $-0,27^{\mathrm{ns}}$ & $0,06^{\mathrm{ns}}$ & $0,02^{\mathrm{ns}}$ \\
\hline FRF_G & $0,20^{\mathrm{ns}}$ & $-0,30^{*}$ & $-0,15^{\mathrm{ns}}$ & $0,20^{\text {ns }}$ & $-0,04^{\mathrm{ns}}$ \\
\hline YF_R & $0,11^{\mathrm{ns}}$ & $-0,03^{\text {ns }}$ & $-0,25^{\mathrm{ns}}$ & $-0,41^{*}$ & $0,10^{\text {ns }}$ \\
\hline RF_R & $-0,04^{\text {ns }}$ & $-0,13^{\text {ns }}$ & $-0,44^{*}$ & $-0,16^{\mathrm{ns}}$ & $0,03^{\text {ns }}$ \\
\hline FRF_R & $-0,02^{\mathrm{ns}}$ & $-0,21^{\text {ns }}$ & $-0,29^{*}$ & $-0,03^{\text {ns }}$ & $0,05^{\text {ns }}$ \\
\hline SFR_G & $0,08^{\text {ns }}$ & $-0,13^{\text {ns }}$ & $0,22^{\mathrm{ns}}$ & $0,20^{\text {ns }}$ & $-0,08^{\text {ns }}$ \\
\hline SFR_R & $0,06^{\mathrm{ns}}$ & $-0,11^{\text {ns }}$ & $0,22^{\text {ns }}$ & $0,16^{\mathrm{ns}}$ & $0,05^{\text {ns }}$ \\
\hline BRR_FRF & $-0,25^{\text {ns }}$ & $-0,30^{*}$ & $-0,38^{*}$ & $-0,61^{*}$ & $-0,18^{\text {ns }}$ \\
\hline FER_RUV & $-0,23^{\text {ns }}$ & $-0,38^{*}$ & $-0,44 *$ & $-0,44 *$ & $-0,19^{\text {ns }}$ \\
\hline FLAV & $-0,22^{\text {ns }}$ & $-0,37 *$ & $-0,45^{*}$ & $-0,43^{*}$ & $-0,17^{\mathrm{ns}}$ \\
\hline FER_RG & $-0,38^{*}$ & $0,10^{\mathrm{ns}}$ & $-0,31^{*}$ & $-0,42^{*}$ & $0,14^{\mathrm{ns}}$ \\
\hline ANTH & $-0,38^{*}$ & $0,11^{\mathrm{ns}}$ & $-0,30^{*}$ & $-0,43^{*}$ & $0,14^{\mathrm{ns}}$ \\
\hline NBI_G & $0,14^{\text {ns }}$ & $0,37^{*}$ & $0,49 *$ & $0,39 *$ & $0,19^{\mathrm{ns}}$ \\
\hline NBI_R & $0,23^{\text {ns }}$ & $0,26^{\mathrm{ns}}$ & $0,53^{*}$ & $0,45^{*}$ & $0,18^{\mathrm{ns}}$ \\
\hline FERARI & $0,00^{\text {ns }}$ & $0,22^{\mathrm{ns}}$ & 0,28 & $0,04^{\text {ns }}$ & $-0,04^{\text {ns }}$ \\
\hline
\end{tabular}

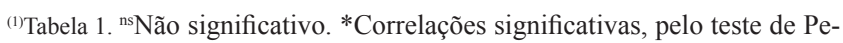
arson, a $5 \%$ de probabilidade $(n=48)$. 
moléculas de clorofila não altera as propriedades de reflectância da cultura (Blackmer et al., 1994), o que reduz as correlações.

Os índices do sensor de fluorescência que melhor explicaram a concentração de $\mathrm{N}_{-} \mathrm{NO}_{3}$ nos pecíolos foram RF_UV, FRF_UV e BRR_FRF, pois apresentaram as maiores correlações. Esses índices têm os UV como fonte de excitação, e a detecção da fluorescência ocorre na faixa do vermelho, vermelho distante e da relação entre amarelo e o vermelho distante. Essas maiores correlações, quando a detecção da fluorescência ocorre na faixa do vermelho, estão vinculadas ao fato de que a fluorescência nessa faixa é emitida somente pela clorofila, e o conteúdo de clorofila nas folhas é conhecidamente relacionado à disponibilidade de $\mathrm{N}$ nas plantas (Schlemmer et al., 2005).

Além destes três índices, outros como o YF_R, FER_RUV, FER_RG, RF_B, NBI_G, NBI_R, FLAV e ANTH foram correlacionados com teor de $\mathrm{N}$ no pecíolo. Segundo Cerovic et al. (1999), além da clorofila, os polifenóis, inclusive as antocianinas (ANTH) e os flavonóis (FLAV) presentes na epiderme, também são afetados pela disponibilidade de $\mathrm{N}$ na planta. Quando em baixa disponibilidade de $\mathrm{N}$, as plantas alocam o excesso de $\mathrm{C}$ na síntese de polifenóis. Portanto, o alto conteúdo de polifenóis pode ser considerado um indicador de baixos teores de $\mathrm{N}$ nas culturas.

Os resultados mais favoráveis aos índices fornecidos pelo sensor de fluorescência corroboram os encontrados por Limbrunner \& Maidl (2007), que observaram boas correlações entre o $\mathrm{N}$ absorvido e as medidas de fluorescência da clorofila em trigo, o que indica que esta ferramenta é eficiente em detectar tanto baixas quanto altas concentrações de $\mathrm{N}$ foliar, sem que ocorra saturação nas curvas, como ocorre com as medidas de reflectância; além disso, mesmo nos estádios iniciais, as correlações foram elevadas, uma vez que somente o material vivo emitiu fluorescência e o solo descoberto não exerceu influência sobre as medidas.

Para mensuração da biomassa de plantas de algodão, além do NDVI, os índices FRF_UV, RF_R, FRF_R, BRR_FRF,FER_RUV,FLAV,FER_RG,ANTH,NBI_G e NBI_R apresentaram correlações significativas (Tabela 4). Tanto o NDVI como os índices do sensor de fluorescência FRF_UV, NBI_G, NBI_R, BRR_FRF, FER_RUV, FLAV e ANTH apresentaram correlação significativa com a produção de biomassa. Além disso, estes índices apresentaram ajuste linear à aplicação de doses crescentes de N (Figuras 2 e 3 ), semelhantemente ao que ocorreu com a biomassa das plantas de algodão. Estes índices apresentam, portanto, capacidade de detectar a mudança na biomassa de plantas de algodão pela aplicação de doses de $\mathrm{N}$.

$\mathrm{O}$ índice que melhor explicou a produtividade foi o índiceclorofila,pois, alémdamaiorcorrelação(Tabela4), apresentouajustequadráticocomasdosesdeN(Figura2), o que também ocorreu com a produtividade (Figura 1). Esses resultados permitem inferir que o índice clorofila apresenta maior capacidade em detectar a variação da produtividade de plantas de algodão do que os demais índices avaliados. Além disso, as doses de $\mathrm{N}$ em que ocorreram a máxima produtividade e o valor máximo do índice clorofila foram próximas: 84 e $78 \mathrm{~kg} \mathrm{ha}^{-1}$ de $\mathrm{N}$, respectivamente. Zhao et al. (2005) afirmaram que a correlação entre produtividade e os índices de
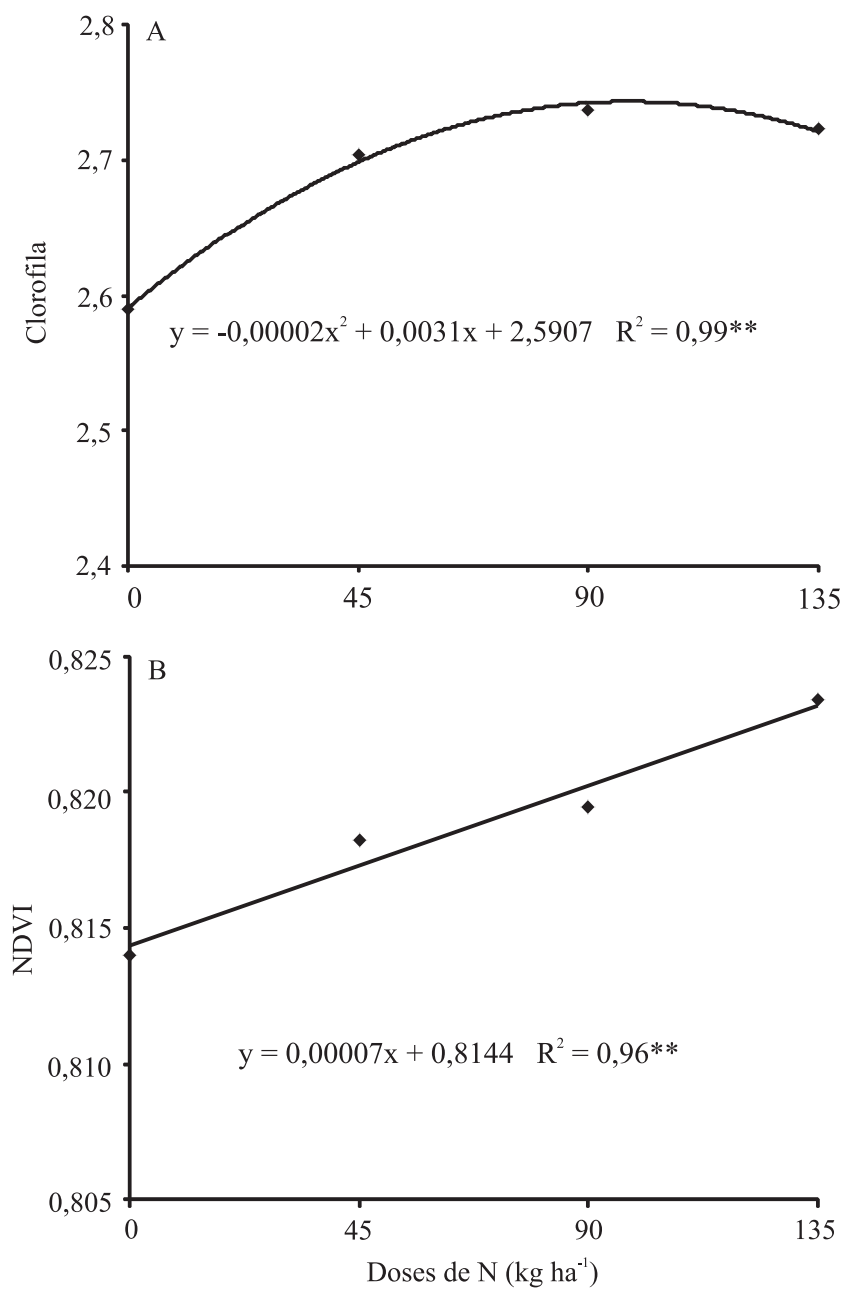

Figura 2. Efeito de doses de N sobre: A, índice do sensor de reflectância NDVI; e B, índice de clorofila. 

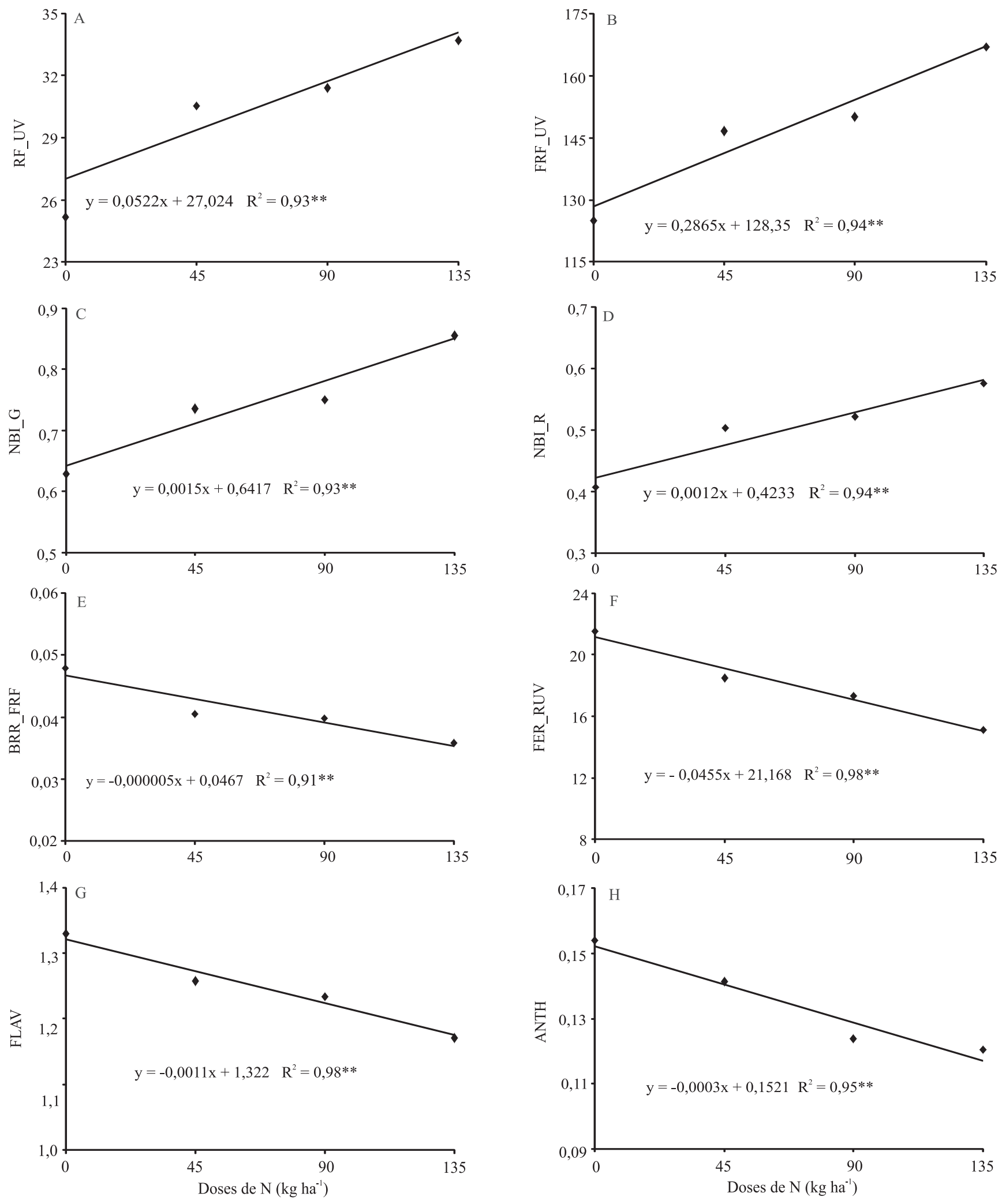

Figura 3. Índices do sensor de fluorescência - RF_UV (A), FRF_UV (B), NBI_G (C), NBI_R (D), BRR_FRF (E), FER_ RUV (F), FLAV (G) e ANTH (H) - em plantas de algodão submetidas a doses de nitrogênio. 
reflectância depende do estádio de crescimento em que as leituras são realizadas e que as mudanças na produtividade de fibras do algodoeiro são fortemente relacionadas aos valores no início da floração.

Além da correlação significativa com os teores de $\mathrm{N}^{-\mathrm{NO}_{3}}$ no pecíolo (Tabela 4), os índices RF_UV, FRF_UV, NBI_G e NBI_R apresentaram relação estatisticamente significativa com as doses de $\mathrm{N}$, tendo-se ajustado por meio de equação de regressão linear positiva. No entanto, os índices BRR_FRF, FER_RUV, FLAV, ANTH ajustaram-se a essa variável por meio de equação linear negativa (Figura 3). $\mathrm{O}$ ajuste linear entre doses de $\mathrm{N}$ e os índices do sensor de fluorescência indica que a dose em que ocorre o valor máximo (linear positiva) ou mínimo (linear negativa) dos índices é superior à dose de $135 \mathrm{~kg} \mathrm{ha}^{-1}$ de N. Porém, quando se observam os teores de $\mathrm{N}_{-} \mathrm{NO}_{3}^{-}$ no pecíolo (Figura 1), verifica-se um incremento linear nos teores de $\mathrm{N}^{-\mathrm{NO}_{3}}{ }^{-}$até aproximadamente $11,07 \mathrm{~g} \mathrm{~kg}^{-1}$ (90 $\mathrm{kg} \mathrm{ha}^{-1}$ de $\mathrm{N}$ ), o que indica que, até este teor, os índices do sensor de fluorescência representam de forma adequada os teores de $\mathrm{N}$ nas plantas de algodão.

Esses resultados são indicativos de que o sensor de fluorescência apresenta potencial para utilização na detecçãode deficiências deNnoalgodoeiro. Limbrunner \& Maidl (2007) observaram boas correlações entre o N absorvido e as medidas de fluorescência da clorofila em trigo. Schächtl et al. (2005) também observaram que o sensor de fluorescência tem capacidade de detectar os teores de $\mathrm{N}$ em plantas de trigo, e Zhang \& Tremblay (2010) observaram o mesmo em plantas de milho.

O sensor de fluorescência utilizado mostrou-se eficiente na detecção dos teores de $\mathrm{N}$ e da biomassa de plantas de algodão, informação que pode ser útil para, combinada com um algoritmo de fertilização, indicar as doses de $\mathrm{N}$ a serem aplicadas, em tempo hábil, no ciclo da cultura. Porém, avaliações de diferentes índices de fluorescência, em laboratório e em campo, na busca de índices mais eficientes para cada estádio, além da inclusão de outras variáveis, tais como horário de coleta, devem ser realizadas para que se verifique o comportamento dos sensores quanto às diferentes intensidades luminosas que podem alterar o comportamento fisiológico das plantas.

A máxima produtividade, estimada com a equação ajustada entre produtividade e doses de N (Figura 1), foi atingida quando aplicados $84 \mathrm{~kg} \mathrm{ha}^{-1} \mathrm{de} \mathrm{N}$. Porém, a máxima eficiência econômica foi encontrada com a aplicação de $56 \mathrm{~kg} \mathrm{ha}^{-1}$ de N. Para os índices do sensor de reflectância, coletados no estádio de emissão do primeiro capulho, os valores na dose de máxima eficiência econômica foram de 0,8183 para o NDVI e 2,702 para o índice clorofila. Quanto aos índices do sensor de fluorescência, os valores foram de: RF_UV, 24,9; FRF_UV, 144,5; NBI_G, 0,726; NBI_R, 0,490; BRR_FRF, 0,0466955; FER_RUV, 18,62; FLAV, 1,260; e ANTH, 0,135. Esses dados servem para indicar o teor ideal para o estádio de emissão do primeiro capulho no algodoeiro.

\section{Conclusões}

1. As variedades de algodão influenciam as leituras dos índices NDVI, CLR, YF_B, RF_B, YF_G, RF_G, FRF_G, FER_RG e ANTH.

2. Os índices do sensor de fluorescência apresentam maior capacidade de detectar os teores de $\mathrm{N}_{-} \mathrm{NO}_{3}^{-}$no pecíolo de plantas de algodão do que os índices do sensor de reflectância.

3. Os índices NDVI, FRF_UV, BRR_FRF, FER_RUV, FLAV, ANTH, NBI_G e NBI_R apresentam maior capacidade de detectar a variação na biomassa de plantas de algodão ocasionada pela aplicação de doses crescentes de $\mathrm{N}$.

4. O índice clorofila, mensurado com o sensor de reflectância, apresenta maior capacidade de detectar a variação da produtividade de plantas de algodão ocasionada pela aplicação de doses crescentes de N.

\section{Agradecimentos}

À Coordenação de Aperfeiçoamento de Pessoal de Nível Superior, por concessão de bolsa. Ao Maricopa Agriculture Center, Maricopa, AZ, EUA, em especial a John Heun, pela ajuda no desenvolvimento deste trabalho.

\section{Referências}

BLACKMER, T.M.; SCHEPERS, J.S.; VARVEL, G.E. Light reflectance compared with other nitrogen stress measurements in corn leaves. Agronomy Journal, v.86, p.934-938, 1994.

BRONSON, K.F.; BOOKER, J.D.; KEELING, J.W.; BOMAN, R.K.; WHEELER, T.A.; LASCANO, R.J.; NICHOLS, R.L. Cotton canopy reflectance at landscape scale as affected by nitrogen fertilization. Agronomy Journal, v.97, p.654-660, 2005.

CEROVIC, Z.G.; SAMSON, G.; MORALES, F.; TREMBLAY, N.; MOYA, I. Ultraviolet-induced fluorescence for plant monitoring: 
present state and prospects. Agronomie: Agriculture and Environment, v.19, p.543-578, 1999.

CISNEROS, J.J.; GODFREY, L.D. Midseason pest status of the cotton aphid (Homoptera: Aphididae) in California cotton: is nitrogen a key factor? Environmental Entomology, v.30, p.501-510, 2001.

FONTANA, D.C.; BERLATO, M.A.; BERGAMASCHI, H. Relação entre o índice de vegetação global e condições hídricas no Rio Grande do Sul. Pesquisa Agropecuária Brasileira, v.33, p.1399-1405, 1998.

FORCE A. User's guide: multiplex: hand-held multi-parameter optical sensor. Available at: < ftp://ftp.dynamax.com/manuals/ Multiplex3_Manual.pdf $>$. Accessed on: 11 Jan. 2010.

GOUlAS, Y.; CEROVIC, Z.G.; CARTELAT, A.; MOYA, I. Dualex: a new instrument for field measurements of epidermal ultraviolet absorbance by chlorophyll fluorescence. Applied Optics, v.43, p.4488-4496, 2004.

HENSLEE, M.A.; MOZAFFARI, M.; SLATON, N.A.; EVANS, E.; KENNEDY, C. Effect of nitrogen fertilizer rate on cotton yield and petiole nitrate concentration. In: SOUTHERN BRANCH OF AMERICAN SOCIETY OF AGRONOMY, 2002. Proceedings. Mobile: American Society of Agronomy, 2002. 22p.

LIMBRUNNER, B.; MAIDL, F.X. Non-contact measurement of the actual nitrogen status of winter wheat canopies by laser-induced chlorophyll fluorescence. In: EUROPEAN CONFERENCE ON PRECISION AGRICULTURE, 6., 2007, Skiathos. Proceedings. Wageningen: Wageningen Academic, 2007. p.173-179.

MA, B.L.; DWYER, L.M.; COSTA, C.; COBER, E.R.; MORRISON, M.J. Early prediction of soybean yield from canopy reflectance measurements. Agronomy Journal, v.93, p.1227-1234, 2001.

MALAVOLTA, E.; VITTI, G.C.; OLIVEIRA, S.A. de. Avaliação do estado nutricional das plantas: princípios e aplicações. 2.ed. Piracicaba: Potafos, 1997. 319p.

MILLER, R.O.; KOTUBY-AMACHER, J. Extractable nitrate, orthophosphate, and chloride of botanical materials: Western States Agricultural Laboratory Exchange Program: suggested soil and plant analytical methods. Version 1.00. [S.1.]: [s.n.], 1994. p.47-48.

MINOLTA. Manual for chlorophyll meter SPAD 502. Osaka: Minolta, 1989. 22p.

MOTOMIYA, A.V. de A.; MOLIN J.P.; CHIAVEGATO E.J. Utilização de sensor óptico ativo para detectar deficiência foliar de nitrogênio em algodoeiro. Revista Brasileira de Engenharia Agrícola e Ambiental, v.13, p.137-145, 2009.

OSBORNE, S.L.; SCHEPERS, J.S.; FRANCIS, D.D.; SCHLEMMER, M.R. Use of spectral radiance to estimate inseason biomass and grain yield in nitrogen- and water-stressed corn. Crop Science, v.42, p.165-171, 2002.

PENNINGTON, D.; TUCKER, T.C. The cotton petiole: a nitrogen fertilization guide. Tucson: University of Arizona, 1984. (Bulletin, 8373).

RAIJ, B. van. Fertilidade do solo e adubação. São Paulo: Agronômica Ceres; Piracicaba: Potafos, 1991. 343p.
RAMBO, L.; SILVA, P.R.F. da; ARGENTA, G.; BAYER, C. Testes de nitrato no solo como indicadores complementares no manejo da adubação nitrogenada em milho. Ciência Rural, v.34, p.1279-1287, 2004.

RAMIREZ, M.B. Monitoring nitrogen levels in the cotton canopy using real-time active-illumination spectral sensing. 2010. 126p. Thesis (Masters) - University of Tennessee, Knoxville.

RAUN, W.R.; SOLIE, J.B.; STONE, M.L.; MARTIN, K.L.; FREEMAN, K.W.; MULLEN, R.W.; ZHANG, H.; SCHEPERS, J.S.; JOHNSON, G.V. Optical sensor-based algorithm for crop nitrogen fertilization. Communications in Soil Science and Plant Analysis, v.36, p.2759-2781, 2005.

REDDY, K.R.; KOTI, S.; DAVIDONIS, G.H.; REDDY, V.R. Agroclimatology: interactive effects of carbon dioxide and nitrogen nutrition on cotton growth, development, yield, and fiber quality. Agronomy Journal, v.96, p.1148-1157, 2004.

SCHÄCHTL, J.; HUBER, G.; MAIDL, F.-X.; STICKSEL, E.; SCHULZ, J.; HASCHBERGER, P. Laser-induced chlorophyll fluorescence measurements for detecting the nitrogen status of wheat (Triticum aestivum L.) canopies. Precision Agriculture, v.6, p.143-156, 2005.

SCHLEMMER, M.R.; FRANCIS, D.D.; SHANAHAN, J.F.; SCHEPERS, J.S. Remotely measuring chlorophyll content in corn leaves with differing nitrogen levels and relative water content. Agronomy Journal, v.97, p.106-112, 2005.

SOLARI, F.; SHANAHAN, J.F.;FERGUSON, R.B.;ADAMCHUK, V.I. An active sensor algorithm for corn nitrogen recommendations based on a chlorophyll meter algorithm. Agronomy Journal, v.102, p.1090-1098, 2010.

STAUT, L.A.; KURIHARA, C.H. Calagem e adubação. In: ALGODÃO: tecnologia de produção. Dourados: Embrapa Agropecuária Oeste, 2001. p.103-123.

UNITED STATES DEPARTMENT OF AGRICULTURE. National Agricultural Statistics Service. Agricultural prices. Available at: $<$ http://usda.mannlib.cornell.edu/MannUsda/viewDocumentInfo. do?documentID=1002>. Accessed on: 25 Dec. 2010.

ZHANG, Y.P.; TREMBLAY,N. Evaluation of the Multiplex fluorescence sensor for the assessment of corn nitrogen status. In: INTERNATIONAL CONFERENCE ON PRECISIONAGRICULTURE, 10., 2010, Denver. Proceedings. Denver: Colorado State University, 2010. 9p.

ZHAO, D.L.; REDDY, K.R.; KAKANI, V.G.; READ, J.J.; CARTER, G.A. Corn (Zea mays L.) growth, leaf pigment concentration, photosynthesis and leaf hyperspectral reflectance properties as affected by nitrogen supply. Plant and Soil, v.257, p.205-217, 2003.

ZHAO, D.L.; REDDY, K.R.; KAKANI, V.G.; READ, J.J.; KOTI, $\mathrm{S}$. Canopy reflectance in cotton for growth assessment and lint yield prediction. European Journal of Agronomy, v.26, p.335-344, 2007.

ZHAO, D.L.; REDDY, K.R.; KAKANI, V.G.; READ, J.J.; KOTI, S. Selection of optimum reflectance ratios for estimating leaf nitrogen and chlorophyll concentrations of field-grown cotton. Agronomy Journal, v.97, p.89-98, 2005.

Recebido em 22 de março de 2012 e aprovado em 23 de julho de 2012 